\title{
ROSSO FIORENTINOS CHRISTUS IN FORMA PIETATIS ZWISCHEN ANDACHT UND SCHÖNHEIT ${ }^{1}$
}

In den letzten Jahrzehnten hat die Geschichte des Körpers und der Sinne eine immense Erweiterung erfahren. Meine Analyse des von Rosso Fiorentino gemalten und heute in Boston aufbewahrten Toten Christus in forma Pietatis (Bild 1) hat jenen Forschungen viel zu verdanken: In der Folge werde ich immer wieder auf die taktilen Qualitäten des Bildes zu sprechen kommen, die es vermögen dem Betrachter eine emotionale Reaktion abzuringen. Meine Interpretation entwickelt sich deshalb entlang folgender Konzepte von Körperlichkeit Tastsinn - Zeit bzw. Zeitpunkt und Einbindung des Betrachters.

Das zwischen Ende 1526 und Anfang 1527 in Rom ausgeführte Bild Rossos konfrontiert uns mit einem fast unversehrten Körper von außergewöhnlicher christlicher Schönheit. ${ }^{2}$ Das Gesicht, welches von dem schulterlang herabfallenden Haar und einem dünnen Bart eingerahmt wird, den ein elegant gezwirbelter Schnauzbart ergänzt, ist bestimmt von einem verzückten Gesichtsausdruck: Die Kontraktionen an den Mundwinkeln entblößen tatsächlich einen Teil des Gebisses, auch wenn dieses Detail in einer Reproduktion kaum zu erkennen ist. Das verzückte Gesicht und der mit an der Oberfläche pulsierenden Gefäßen versetzte Körper - Einzelheiten, die dem Bild taktile Qualitäten von ungewöhnlicher Realitätsnähe verleihen - sind nicht von einander zu trennen.

Rossos Werk ist in vielerlei Hinsicht einzigartig: Nicht nur vom ikonographischen Standpunkt aus, denn der von vier prächtigen Engeln umringte forma Pietatis del Rosso Fiorentino fra devozione e bellezza, in: Christoph L. Frommel/Gerhard Wolf (Hg.): L'immagine di Cristo, Rom 2006, S. 324-340.

2 Die Bibliografie zum Werk Rossos hat sich beträchtlich erweitert. Die wichtigsten Beiträge sind in den folgenden Verweisen zusammengefasst. Für grundlegende Informationen zum Bild kann auf diese Monografie zurückgegriffen werden: David Franklin: Rosso in Italy. The Italian Career of Rosso Fiorentino, New Haven/London 1994, S. 139-148. 


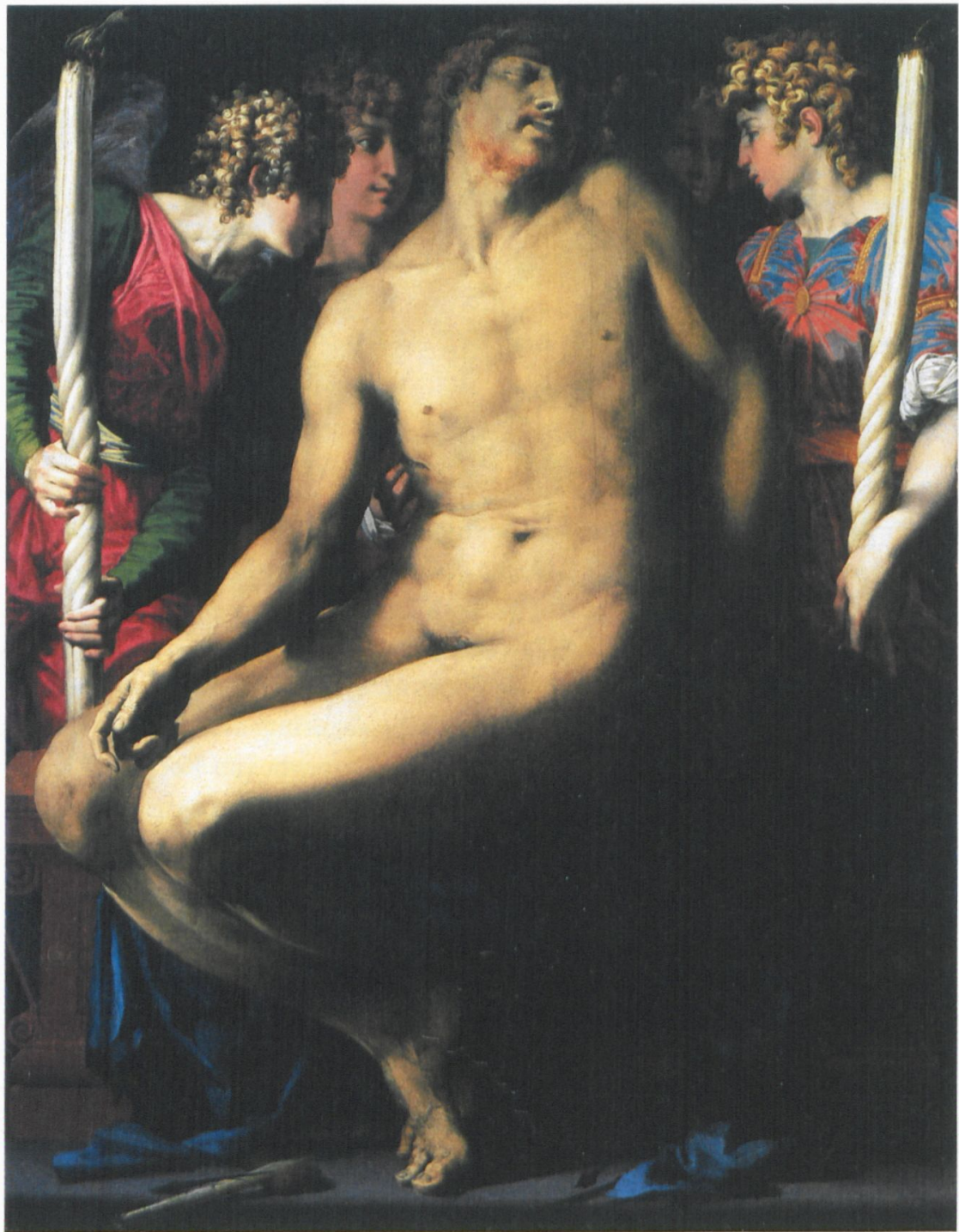

Bild 1 Rosso Fiorentino: Christus in forma Pietatis, 133,5 × 104,1 cm, Museum of Fine Arts, Boston.

Christus ist just in dem Moment wiedergegeben, als er im Halbschatten des Grabes zu neuem Leben erwacht, sondern auch in Bezug auf eine viel weitergehende visuelle Kultur. Bis dahin hatte es außer Rosso kein anderer Künstler gewagt, den Körper Christi völlig nackt und in voller Lebensgröße zu malen. Das Altarbild misst in der Höhe einen Meter und dreiunddreißig Zentimeter, und käme die Figur zum Stehen, würde sie eine beeindruckende Größe erlangen. 
Wie konnte es aber dazu kommen? Die tiefe Religiosität des Bildes kann keinen Augenblick bezweifelt werden - und was sollte ein Christus „in forma pietatis", der in der ersten Hälfte des Cinquecento in Rom gemalt wurde, anderes verkünden ${ }^{3}$ Doch an dieser Stelle ist es nicht so sehr von Interesse, nach dem Was und dem Warum zu fragen, als vielmehr das Wie zu erkunden. Unter diesem Aspekt scheint es mir durchaus berechtigt, davon auszugehen, dass nur wenige Betrachter die Beklemmung zu unterdrücken vermöchten, die von dieser Darstellung ausgeht - ein Missbehagen, das zum Teil von den leicht geöffneten Lippen Christi und der Geste des jünglinghaften Engels herrührt, der mit dem Druck seiner Finger die Seitenwunde erneut öffnet. Selbstverständlich muss man zwischen unserer Wahrnehmung und jener frühneuzeitlichen unterscheiden, doch muss man sich dabei auch fragen, welche Kultur das Aufkommen eines so eigenartigen Andachtsbildes begünstigt haben könnte und ob das Gemälde seinerzeit auch eine solche Irritation auslöste - oder ob es diese Wirkung nur auf uns heutzutage hat.

Mit diesem Unbehagen konfrontiert, können wir nichts anderes tun, als mit den herkömmlichen Mitteln des Fachs zu reagieren, das heißt die Texte und Kontexte zu erörtern, um eine erste gewiss oberflächliche Lektüre des Bildes zu korrigieren, und auf kulturelle Modelle zurückzugreifen, die seinerzeit akzeptiert waren. Angesichts des Unbehagens, das dieses Bild hervorruft, schützen wir uns also mit den Texten der Theologen und mit der Nachahmung der Antike. Wir werden uns nicht die Frage stellen, wie Rosso auf die Idee gekommen sein könnte, ein verzücktes Gesicht und einen schönen, pulsierenden und fühlbaren Körper darzustellen, sondern stattdessen die Frömmigkeit erforschen, die in diesem Bild ausgedrückt, widergespiegelt oder hervorgerufen wird.

In der Vergangenheit wurde das Bostoner Bild auf unterschiedlichste Art kontextualisiert. Manche sahen darin einen Anklang an Savonarolas Trattato dell'amore di Gesù Cristo. ${ }^{4}$ Einige einen sehr orthodoxen Ausdruck theatiner Frömmigkeit mit antilutherischen Konnotationen. ${ }^{5}$ Andere interpretierten Ros-

Die Vollmacht vom 29. September 1527, ausgehändigt vom Maler an einen gewissen Lodovico Bruschi da Reggio, autorisiert diesen, die, aufgrund des Sacco (di Roma) in Rom hinterlassene Tafel wieder herzustellen, die von Rosso selbst beschrieben wird als: „unum quadrum lignaminis, ornatum picturis, et in quo inest picta figura domini nostri Iesu Christi in forma Pietatis"; siehe auch: David Franklin: New Documents for Rosso Fiorentino in Sansepolcro, in: Burlington Magazine 131 (1989), S. 817-827 u. insb. S. 822 u. 826f., Dokument 5.

4 Carlo Falciani: Intorno alla committenza Appiani: $l^{\prime}$ „immaginazione“ della morte di Cristo nelle opere del Rosso Fiorentino, in: Roberto Paolo Ciardi/Antonio Natali (Hg.): Pontormo e Rosso. Atti del convegno di Empoli e Volterra. Progetto Appiani di Piombino, Venedig 1996, S. 272 f.

5 Philippe Costamagna: La création de l'ordre des Théatins et ses répercussions sur l'art de Rosso Fiorentino et de ses contemporains, in: Ciardi/Natali (Hg.): Pontormo 
sos Bild im Kontext der eucharistischen Theologie des Tommaso de Vio als Erwiderung auf die Anschuldigungen Zwinglis. ${ }^{6}$ Nicht weniger überzeugend sind die Argumente jener, die das Gemälde in das „spirituelle" Ambiente um Vittoria Colonna stellen, wobei sie sich auf den folgenden Passus der Pianto della Marchesa di Pescara sopra la Passione di Cristo beziehen: "la bruttezza de la morte era non solo bella nel bellissimo volto ma la fierezza se convertí in dolcezza grande, la oscurità in chiara luce. ${ }^{77}$ Und man könnte noch die Überlegungen O'Malleys anfügen, der sich im postscriptum von Steinbergs Buch über die Sexualität Christi fragte, ob die in der franziskanischen Frömmigkeit des Spätmittelalters beliebte und auch zu Beginn des Cinquecento noch aktuelle Redewendung Nudum sequi nudum Christum nicht zum Teil die allgegenwärtige „Man of Sorrows"-Ikonographie in der Renaissance rechtfertigen könnte. ${ }^{8}$

Zweifellos steckt in allen diesen Vorschlägen etwas Wahres oder Wahrscheinliches, doch bleiben einige der Thesen bestenfalls hypothetisch, solange wir nicht mit Sicherheit wissen, wer das Werk in Auftrag gegeben hat (wahrscheinlich Bischof Leonardo Tornabuoni, ab 1522 Bischof von San Sepolcro) ${ }^{9}$

e Rosso (wie Anm.4), S.157-163, welcher den "theatinischen" Weg wieder aufnimmt und weiterentwickelt, wie er bei Hartt angelegt war: Frederick Hartt: Power and the Individual in Mannerist Art, in: The Renaissance and Mannerism. Studies in Western Art. Acts of Twentieth International Congress of the History of Art, II, New York, 7.-12. September 1961, Princeton 1963, S. 231.

6 Regina Stefaniak: Replicating Mysteries of the Passion. Rosso's Dead Christ with Angels, in: Renaissance Quarterly 45 (1992), S. 677-738.

7 Alberto Mugnaini: Feritas, humanitas, divinitas nell'opera del Rosso. Il problema degli influssi religiosi, letterari e scientifici, in: Ciardi/Natali (Hg.): Pontormo e Rosso (wie Anm. 4), Venedig 1996, S. 133.

8 John W. O'Malley, S. J.: Postscript, in: Leo Steinberg: The Sexuality of Christ in Renaissance Art and in Modern Oblivion, New York 1983, S. 199-203 u. insb. S. 202.

9 Als Auftraggeber des Werkes galt bislang Leonardo di Lorenzo Tornabuoni, obgleich in Vasaris Viten überliefert ist, dass ein toter Christus von zwei und nicht von vier Engeln gestützt wird: Giorgio Vasari: Le Vite de' più eccellenti pittori, scultori e architettori nelle redazioni del 1550 e 1568, 6 Bd., Bd. 4, hg. v. Rosanna Bettarini/Paola Barocchi, Florenz 1966-1987, S. 481. Weitere Indizien scheinen die Identität jenes Gemäldes zu bestätigen, welches von Vasari im römischen Haus des Monsignor Della Casa, dem Erbe Tornabuonis, oder einer seiner Nachfahren, gesehen worden ist, mit der heute in Boston befindlichen Tafel. Allerdings sind diese Indizien nicht ausreichend, um zu zeigen, dass das Gemälde, das heute in den Vereinigten Staaten ist, vom Bischof in Auftrag gegeben wurde, auch wenn dies die wahrscheinlichste Hypothese bleibt. Dennoch konnte bislang nicht geklärt werden, weshalb der Auftraggeber das Gemälde nicht mit sich in die Toskana gebracht hatte, wenn dieses tatsächlich für die Ausschmückung einer Kapelle vorgesehen war, die von ihm gegründet worden war. Der Auffassung von André Chastel: Il sacco di Roma, Torino 1983, S. 149, zufolge hielt sich der Bischof während der Plünderung in Rom auf. Wenn das Gemälde ihm gehört hätte, so hätte er es nach Sansepolcro mitnehmen können, als er die Stadt verließ. Warum tat er dies nicht? Das Gemälde hat moderate Ausmaße, aber in den chaotischen Tagen des Sacco konnte 
und welche genaue Funktion ihm ursprünglich zugedacht war, obwohl das Gemälde sicherlich als Altarbild geplant war. Angesichts der Fülle von Bezügen kommen einem die monita Leo Steinbergs in den Sinn, mit denen er sich gegen eine Tendenz der Interpretation wandte, die er selbst als "textism" bezeichnete und der man einen ebenso diffusen „contextualism“ hinzufügen könnte. Darüber hinaus könnte man eine Passage aus John Shearmans Buch über die Wandteppiche in der Sixtinischen Kapelle zitieren: "The iconography of [Raphael's] tapestries has no positive tendency that can be isolated as Augustinian, Dominican or Franciscan [...]. ${ }^{\text {10 }}$ Die grundlegenden Ideen waren vielmehr im Umlauf und gehörten zum Allgemeingut. Bezogen auf unser Problem ergibt sich daraus die folgende Frage: Könnte es nicht sein, dass Rosso Fiorentino von sich aus ein Werk geschaffen hat, das ungewollt ambivalent ist?

An die Theologen geht die Aufgabe, die ungewöhnlichen Aspekte des Gemäldes zu deuten. Die Passionssymbole sind augenfällig: der Schwamm auf der Spitze der gebrochenen Lanze, die drei Nägel und der königliche Mantel, die ostentatio vulnerum, jenes Opferzeichen, das für unsere Errettung und für die Furcht vor dem Tod steht, und das, übertragen auf die Schönheit des nackten

auch ein relativ kleines Objekt wie dieses sperrig sein. Es ist auch möglich, dass das Gemälde noch nicht fertig war. Dennoch ist nicht klar, warum der Bischof nicht versuchte, zurückzukehren oder nach dem Sacco wieder in den Besitz des Gemäldes zu gelangen. Wenn das Werk von Tornabuoni in Auftrag gegeben wurde, warum hätte Rosso nicht einfach seine Kontakte in Rom nutzen können? Warum jemanden Unbekannten beauftragen, um mit den Franziskaner-Nonnen von San Lorenzo in Panisperna zu verhandeln, wo der Künstler sein Gemälde aufbewahrt hielt. Wäre es nicht logischer gewesen, direkt den mächtigen Prälaten zu involvieren, dem das Gemälde ja auch theoretisch zustand? Wenn wir versuchen eine Antwort auf diese Fragen zu finden, so geht die Sicherheit, mit der der Bischof Tornabuoni als Auftraggeber des Christus "in forma pietatis" benannt wird, verloren. Es scheint hingegen möglich zu sein, dass Rosso sich die Tafel nach Sansepolcro hat zuschicken lassen, weil er es an den Bischof hätte verkaufen können, auch weil die Ikonographie des Gemäldes sich in glücklicher Koinzidenz mit dem Titel seiner Diozöse befand. Wenn sich diese Überlegungen bewahrheiten sollten, so müsste nach einer möglichen Alternative zu Tornabuoni als Auftraggeber gesucht werden, einer Person, die möglicherweise im Sommer 1527 verstorben ist. Auch wenn keine unzweideutigen Beweise zur Stützung dieser Hypothese gefunden werden konnten, so lässt sich zumindest ein Kandidat benennen: Eubel zufolge wurde der Bischof von Lucera Domenico de' Jacobacci, ein fähiger Rechtsgelehrter und Theologe, am 6. Juli 1517 zum Kardinal mit dem Titel von San Lorenzo in Panisperna gewählt; es ist wahr, dass Jacobacci wenige Tage später auf einen anderen Posten versetzt wurde, aber Charles Berton: Dictionnaire des Cardinaux, Paris 1857, col. 1099, teilt uns mit, dass Jacobacci am 2. Juli 1527 starb. Zu einer Biografie des Kardinals, einem der Protagonisten des 5. Lateranischen Konzils, siehe Josef Klotzner: Kardinal Dominicus Jacobazzi und sein Konzilswerk, Rom 1948. and the Tapestries for the Sistine Chapel, London 1972, S. 90. 
Körpers des Heilands, zu einem Versprechen der Erlösung wird. Doch bilden die greifbare Realität dieses nackten Körpers und das verzückte Antlitz eigentlich das aufsehenerregendste Motiv des Bildes, das häufig zu sehr an den Rand des ikonographisch-theologischen Diskurses gerückt wird. Zweifellos tauchen in den Analysen der Tafel die sinnlichen, erotischen und sogar androgynen Aspekte immer wieder auf, doch hat nur Regina Stefaniak auf die laszive Natur der Darstellung, die geradezu anrüchige Pose und den außerordentlichen erotischen Einschlag des Bildes hingewiesen, ganz zu schweigen von den "phallischen Symbolen" und der mutmaßlich weiblichen Natur des auferstanden Christus. ${ }^{11}$ Ich bin nicht davon überzeugt, dass diese Begriffe dazu geeignet sind, die Ambivalenz des Bildes zu erklären, doch haben sie dankenswerterweise ans Licht gebracht, was andere verschwiegen oder nur verlegen angedeutet haben. Stefaniak irrt, doch hat sie den Finger in die Wunde gelegt.

Ich schlage deshalb vor, zwei Richtungen weiter zu verfolgen. Erstens: Auch wenn der Begriff Engelpietà eine Erfindung der Kunstgeschichte ist und Rossos Bild eine ikonographische Besonderheit darstellt, kann es hilfreich sein, das Gemälde ähnlichen Bildern gegenüberzustellen, um seine taktilen Qualitäten hervorzuheben und letztlich nachzuvollziehen, was den modernen Betrachter so irritiert. ${ }^{12}$ Zweitens möchte ich in diesem Kontext die Begriffe bellezza und decoro diskutieren, was bei der Definition von möglichen Rezeptionsebenen des Gemäldes im Hinblick auf Rossos Zeitgenossen von Nutzen sein kann.

\section{Engelpietà}

Was den ersten Punkt betrifft, so ist die von Engeln flankierte Imago pietatis spätestens seit Beginn des Trecento in Italien bekannt, insbesondere seit der Zeit von Giovanni Pisanos Kanzel, heute in Berlin. Weite Verbreitung fand sie vor allem seit dem vierten Jahrzehnt des Quattrocento und mit besonderer Intensität seit der Mitte des 15. Jahrhunderts, ${ }^{13}$ nicht zuletzt weil das Mantuae sanguis preciosus anlässlich des von Pius II. 1459 einberufenen Reichstages zur Abwehr

13 Andrea de Marchi: Un raggio di luce su Filippo Lippi a Padova, in: Nuovi Studi. Rivista di arte antica e moderna 1 (1996), S. 10f. und 19, Anm. 35. Siehe ebd.: Centralità di Padova: alcuni esempi di interferenza tra scultura e pittura nell'area adriatica alla metà del Quattrocento, in: Quattrocento Adriatico. Fifteenth-Century Art of the Adriatic Rim. Papers from a Colloquium held at the Villa Spelman, Florenz 1994, hg. v. Charles Dempsey, Bologna 1996, S. 71-76. 


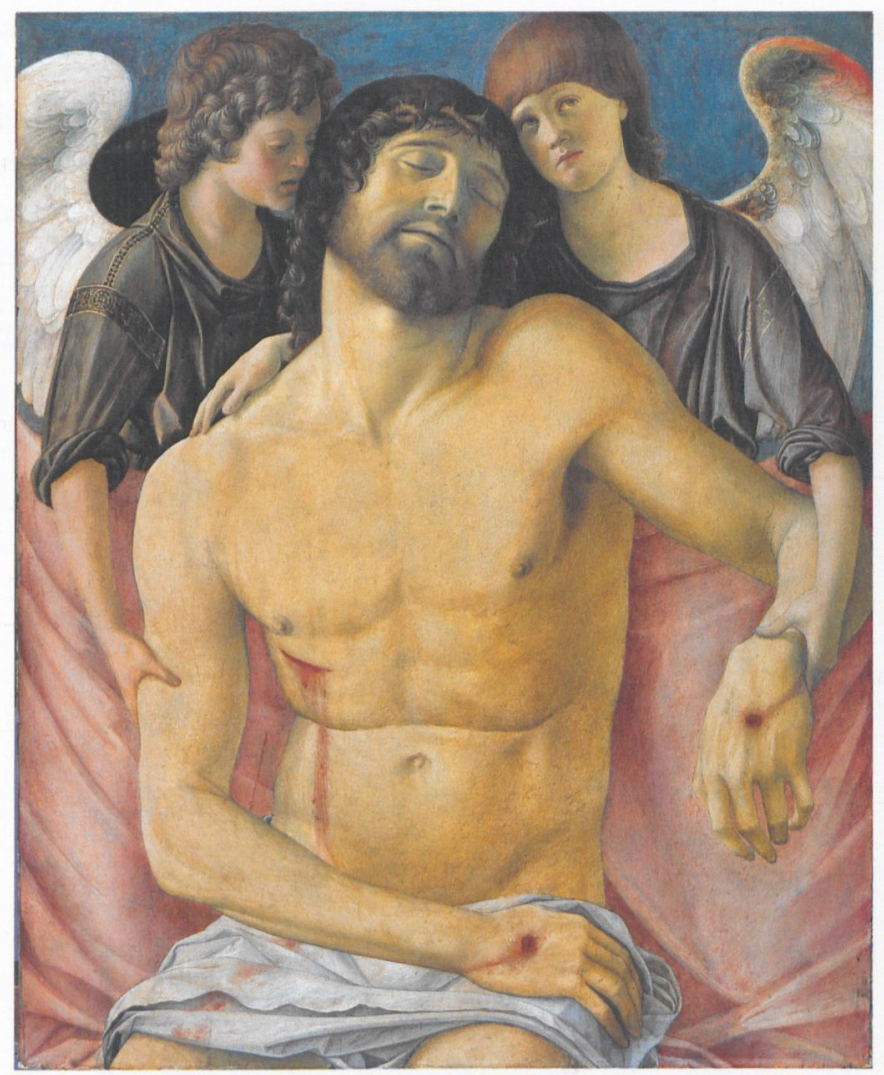

Bild 2 Giovanni Bellini: Der tote Christus von zwei Engeln gestützt, 1475-1478, Gemälde, Pappelholz, Gemäldegalerie (SMB), Berlin.

der türkischen Gefahr zum erneuerten Kultobjekt wurde. ${ }^{14}$ Nach dem wiederbelebten Kult des Mantuae sanguis preciosus nehmen die Beispiele in der zweiten Hälfte des Quattrocento in großem Maß zu, vor allem in Norditalien, angefangen bei Carlo Crivelli ${ }^{15}$ und Giovanni Bellini (Bild 2) bis Antonello da Messina und Francesco Francia ${ }^{16}$.

Die Serie bringt uns zu folgenden Resultaten. Erstens: Ungeachtet der Funktion der Bilder, ist Christus immer mit dem Lendentuch oder dem Schweiß-

Andreas Hauser: Andrea Mantegnas ,Pietà'. Ein ikonoklastisches Andachtsbild, in: Zeitschrift für Kunstgeschichte 63 (2000), S. 455.

15 Martin Davies: The Earlier Italian Schools, London 1986, S. 153-156.

16 Andrea Emiliani/Konrad Oberhuber: Bologna 1490. Dall'umanesimo severo alla suavitas rinascimentale, in: Marzia Faietti/Konrad Oberhuber (Hg.): Bologna e I'umanesimo 1490-1510, Bologna 1988, S. XII. Emilio Negro/Nicosetta Roio: Francesco Francia e la sua scuola, Modena 1998, S. $134 \mathrm{f}$. 


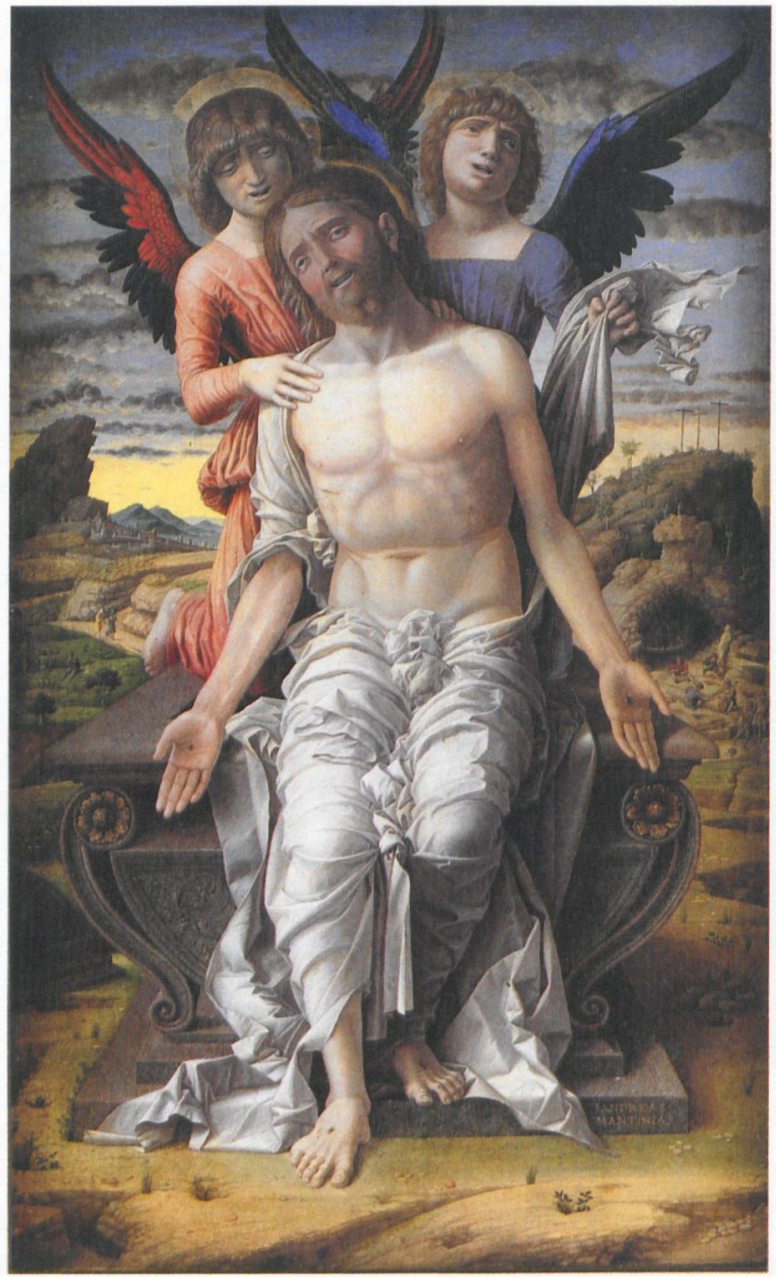

Bild 3 Andrea Mantegna: Schmerzensmann mit zwei Engeln, 1500, Tempera/Holz, $78 \times 48 \mathrm{~cm}$, Statens Museum for Kunst, Kopenhagen.

tuch bedeckt. Zweitens: Die Engel sind fast immer als Knaben, kleine Jungen, gegeben und selbst wenn sie als Jünglinge erscheinen und mit der Haut des Erlösers in direktem Kontakt kommen, wie bei der Tafel von Giovanni Bellini in Berlin, berühren sie niemals die Seitenwunde. Niemals reißen sie die Wunde auf, wie es der von Rosso gemalte androgyne Engel tut.

Auch wenn das Werk Mantegnas, das sich heute in Kopenhagen befindet (Bild 3), nur 83 auf 51 Zentimeter misst (genau 50 Zentimeter weniger als Rossos Gemälde), steht es in gewisser Hinsicht der Version des Florentiner Malers 
sehr nah. ${ }^{17}$ Wie bei dem Bostoner Bild ist die Signatur unten rechts angebracht, der athletische Körper Christi ist ganzfigurig, auf einem Sarkophag sitzend dargestellt, und die beiden Engel sind jünglingshaft. Darüber hinaus haben wir es auch in diesem Fall mit einer Hybridform von symbolischem und narrativem Bild zu tun, denn während der Bildvordergrund durch die Pietà bestimmt wird, sind im Hintergrund links zwei Marien zu erkennen, die sich zum Grab begeben. Dabei provoziert Mantegnas Werk eine andere Art von Beklemmung:

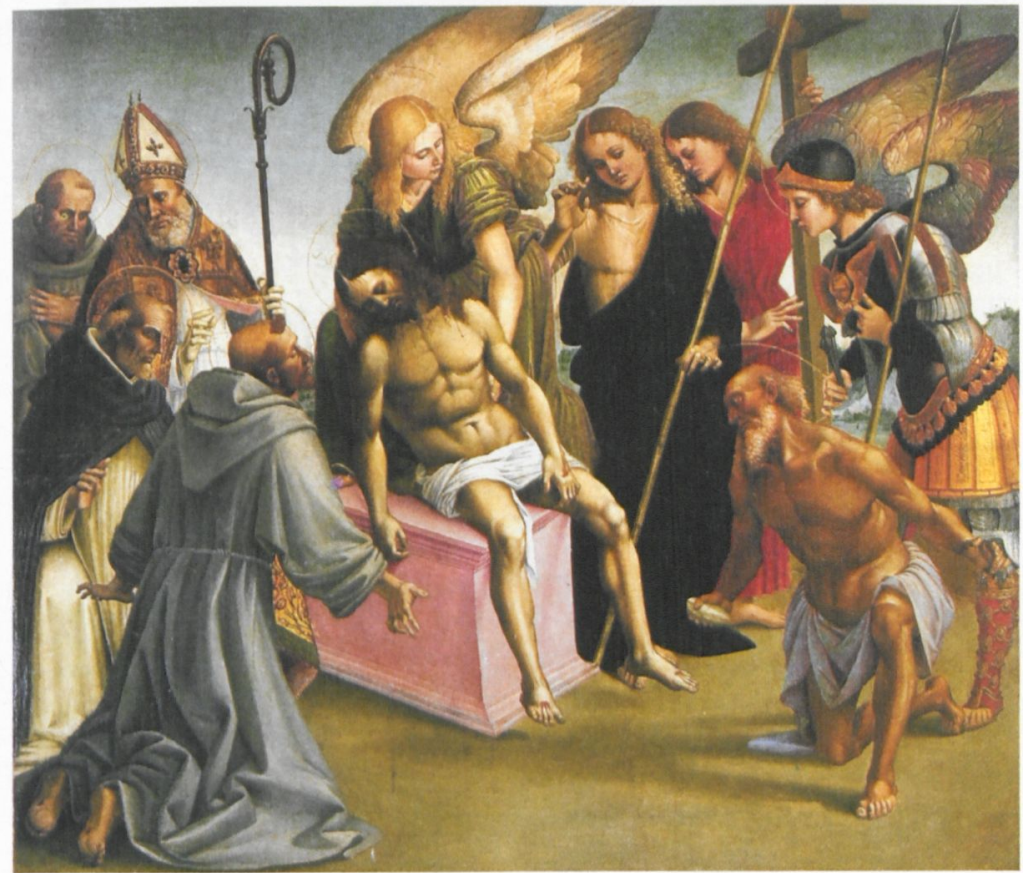

Bild 4 Signorelli: Pietà und Heilige, um 1507-1510, Öl auf Holz, 153 × 182 cm, San Niccolò, Cortona.

Obgleich einer der auf dem Sarkophag knienden Engel die Haut des Erlösers mit den Händen streift, geht er nicht so weit, die Wunde wieder aufzureißen. Christus Miene deutet den erlittenen Schmerz, nicht aber die Ekstase an. Sie ist voller Aktivität und zieht unsere Aufmerksamkeit auf sich, so dass wir nicht „verkönnte man sogar annehmen, dass Rosso es gekannt haben könnte. Das Gemälde ist zum ersten Mal im Inventar des Kardinal Silvio Valenti in Rom erwähnt worden; siehe die Liste bei Keith Christiansen, in: Jane Martineau (Hg.): Andrea Mantegna, Mailand 1992, S. 244. 


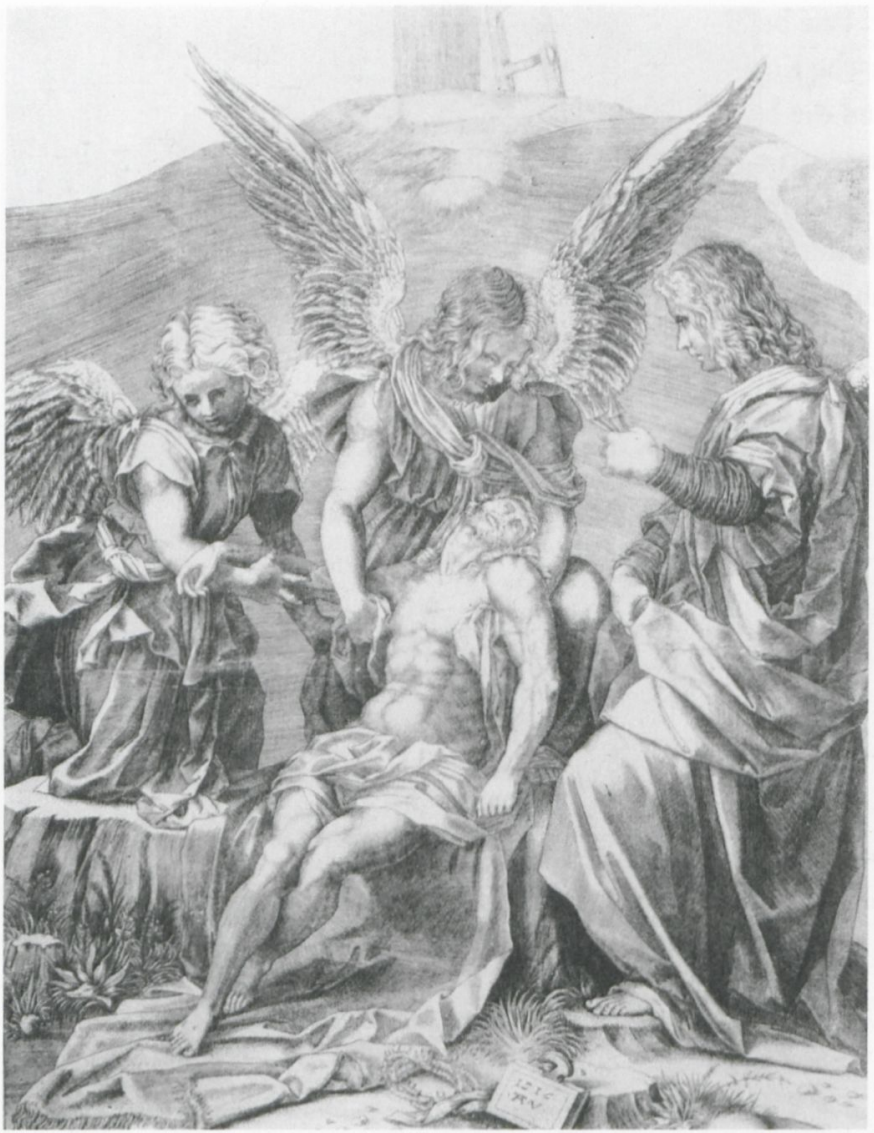

Bild 5 Agostino Veneziano (nach Andrea del Sarto): Puccini Pietà, Kupferstich, Metropolitan Museum of Art, New York.

gnüglich" bei seinem Brustkorb verweilen können, wie wir es dagegen bei Rossos Christus tun können. Im Unterschied zu diesem ist in Mantegnas Werk die Morgendämmerung bereits angebrochen und taucht die Landschaft in ein Licht, das der Szene einen Anklang von Zuversicht verleiht. Vor allem aber ist der Salvator nicht in völliger Nacktheit wiedergegeben. Wohl deshalb hat man sich bei der Analyse des von Mantegna geschaffenen Leidensbildes nie auf die Konzepte von Sexualität und Erotik berufen.

Wenn wir zu den toskanischen Beispielen übergehen, die Rosso gekannt haben könnte, etwa Signorellis Altarbild in San Nicolò in Cortona (Bild 4) oder die von Andrea del Sarto gemalte und heute verlorene Pietà Puccini ${ }^{18}$ (Bild 5) 
fallen die wesentlichen Unterschiede ins Auge. Zwar stellte auch Signorelli jünglingshafte Engel dar, doch distanzierte er sie vom Körper Christi; bei Andrea del Sartos Altarbild spielt sich die Szene hingegen vor offener Landschaft, am Fuße des Kalvarienberges ab und nicht am Grab. Der Sarkophag ist hier nicht vorhanden.

Fassen wir also zusammen. Die ikonographischen Varianten dieses Typus sind so zahlreich, dass man sich fragen muss, ob die sogenannte Engelpietà tatsächlich existierte. Zudem gibt es auch für die Gemälde von Andrea del Sarto und Mantegna keine unmittelbaren Vergleiche, doch vermittelt uns, den heutigen Betrachtern, erst Rossos Werk ein gewisses Gefühl von Beklommenheit. Dieses Missbehagen wird mindestens durch fünf Faktoren verursacht:

1. Nur hier wird der muskulöse Körper Christi in der Malerei in voller Lebensgröße und in völliger Nacktheit wiedergegeben.

2. Die Masse eines „realen” Körpers, der auf einer reduzierten Fläche zusammengedrängt ist, erzeugt zudem eine Art Klaustrophobie: es entsteht der Eindruck, als befände man sich in Angesicht zu Angesicht mit dem Erlöser auf engem Raum im Inneren des Grabes. Die offenen und Ruhe ausstrahlenden Landschaftshintergründe Andrea del Sartos und Mantegnas fehlen.

3. Keiner der Abgebildeten sucht den Blickkontakt zum Betrachter, so dass wir unmittelbar mit dem nackten Körper konfrontiert sind. In diesem Fall rührt das Unbehagen von dem Umstand her, dass sich uns das Bild gleichsam passiv darbietet.

4. Auch wenn die Engel geschlechtlos sind, gibt es einen wesentlichen Unterschied zwischen den Knaben, die Donatello, Crivelli oder Francia geschaffen haben, und den Jünglingen Rossos. Einer davon streift nicht die Haut des Erlösers, sondern betastet die Seitenwunde.

5. Auf dem Gesicht Christi zeigt sich eine gewisse Verzückung. ${ }^{19}$

\section{bellezza und decor}

Wie kann man diese neue fleischliche Schönheit erklären? Der locus classicus hinsichtlich der äußeren Erscheinung Christi ist der Psalm 45(44), 3: „Speciosus forma prae filiis humanis“, „Du sollst schön sein, der Schönste unter den menschsehen werden; siehe in diesem Zusammenhang: Giovan Battista Armenini: De' veri precetti della pittura, hg. v. Marina Gorreri, m. e. Vorw. v. Enrico Castelnuovo, Torino 1988, S. 191. 


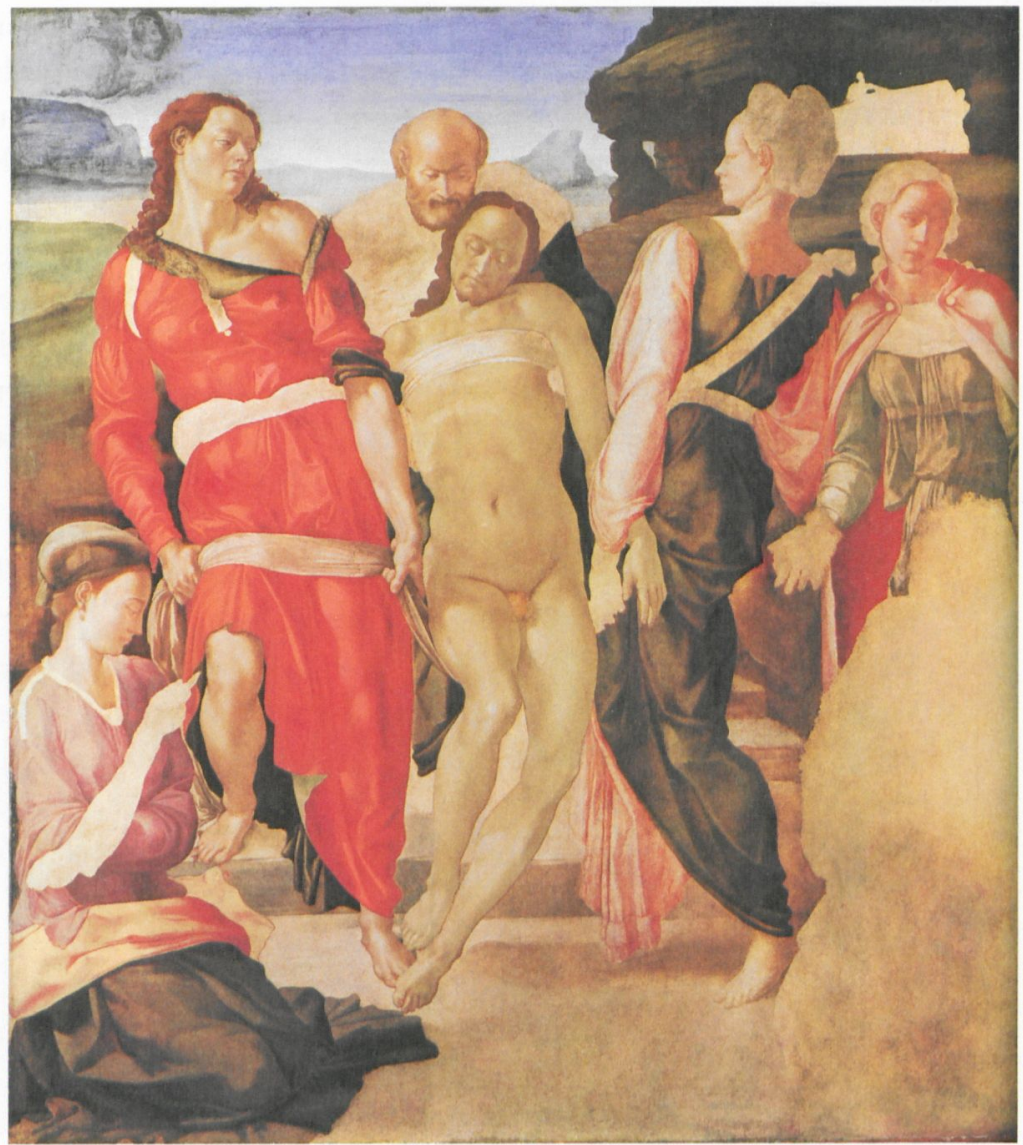

Bild 6 Michelangelo: Grablegung Christi, um 1501, Tempera und Öl auf Holz, $159 \times 149 \mathrm{~cm}$, National Gallery, London.

lichen Nachkommen: auf deinen Lippen liegt ein anmutiger Zauber", eine Auffassung, die die Theologen um 1500 teilten. ${ }^{20}$

Auch in den Schriften Albrecht Dürers, die einige Jahre vor Rossos Gemälde in Umlauf waren, taucht das Lob auf die Schönheit des Erlösers wieder auf, etwa in jenem Passus, in dem der Künstler fordert, daß beim Malen der Figur Christi dieselben Kriterien zugrunde gelegt werden sollten wie jene, die die von Plinius gelobten griechischen Künstler bei der Darstellung der Schönheit des Gottes Apoll anwandten: „Dann zu gleicher Weis, wie sie die schönsten Gestalt eines Menschen Anm. 5. 


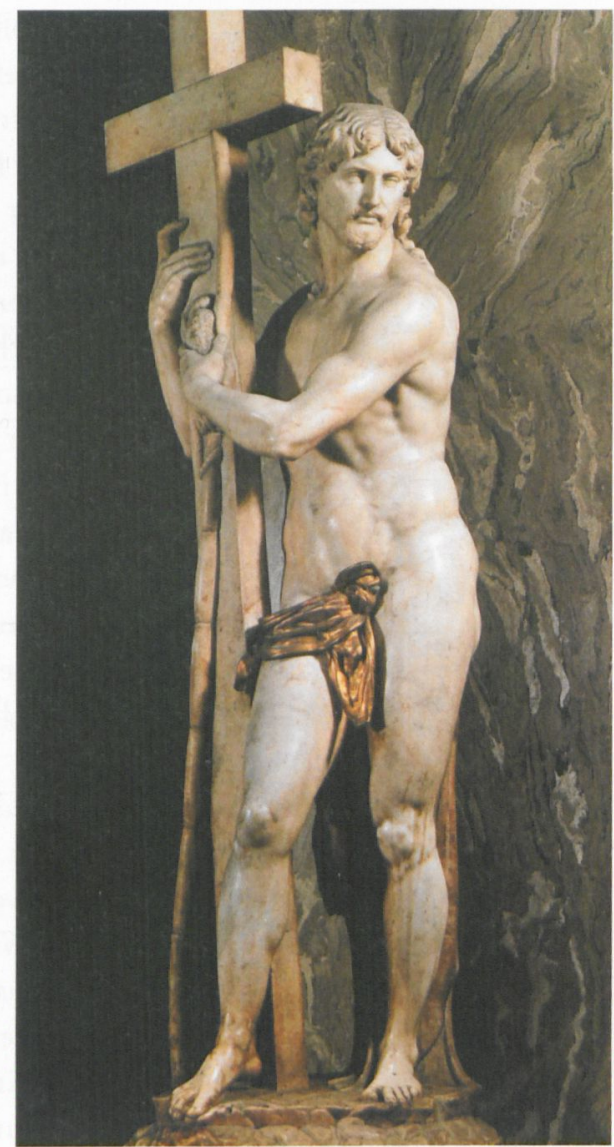

Bild 7 Michelangelo: Auferstandener Christus, um 1518/1520, Marmor, 250 cm, Santa Maria sopra Minerva, Rom.

haben zugemessen ihrem Abgott Apollo, also wollen wir dieselb Moss brauchen zu Crysto dem Herren, der der schönste aller Welt ist." ${ }^{\text {21 }}$

Diese Eindrücke verhallen jedoch in der Stille oder bleiben unvollständig, solange wir nicht die visuelle Kultur jener Zeit in Erwägung ziehen. Dabei ist der Rekurs auf die Modelle Michelangelos und jene der Antike nicht hinreichend, wenn er allein als ein ikonographisches Schema oder formales und gelehrtes Zitat verstanden wird.

Die Unterschiede sind in der Tat von größerer Bedeutung als die Analogien. Michelangelo bleibt für Rossos Bild ein unumgänglicher Referenzpunkt, 
doch stellt der für Santo Spirito geschaffene Gekreuzigte, wenngleich vollkommen nackt, eine Ausnahme dar und ist nicht lebensgroß. Der Christus der vatikanischen Pietà ist mit einem Lendentuch bedeckt. Der zum Grab getragene Christus (Bild 6), der einzige wirkliche Vorgänger von Rossos Gemälde, ist bezeichnenderweise unvollendet geblieben, vor allem aber zeigt der Körper kaum Anzeichen von Gewalt. Hände und Füße weisen keine Wunden auf, 22 nur unterhalb der Brustbinde öffnet sich eine kleine Risswunde, doch ist diese sicher nicht mit dem fast wollüstigen Schnitt im Fleisch von Rossos Figur zu vergleichen.

Auch andere Modelle Michelangelos reichen nicht aus, um die besondere Komposition des Bostoner Gemäldes näher zu beleuchten. Die ignudi der Sixtinischen Decke, die zu Recht als formale Inspirationsquellen von Rossos invenzione bezeichnet wurden, stellen nicht Christus dar. Dasselbe könnte man von dem sterbenden Sklaven sagen. Der auferstandene Christus in der Minerva-Kirche (Bild 7) ist hingegen nicht im Zustand der Erschlafftheit dargestellt, sondern in jenem der Vitalität: Sein heroischer Körper gibt sich nicht passiv einer ekstatischen Pose hin, sondern schreitet bei vollem Bewusstsein und in vollkommener Autonomie einher.

Auch Verweise auf die antike Skulptur dienen, obwohl legitim, lediglich dazu, die beängstigende Einzigartigkeit von Rossos Bild zu verschleiern. Zwar könnte sich der Künstler am Bett Poliklets inspiriert haben, doch hat er von der antiken Bildhauerei und den Skulpturen Michelangelos vor allem den Wunsch assimiliert, den Körper plastisch wiederzugeben - nicht im Sinne eines unmittelbaren Zitates, sondern vielmehr hinsichtlich der Konzeption des Körpers.

Für Rossos Christus wurden häufig die Begriffe erotisch und sinnlich verwendet, doch welche Bedeutung hatten diese Begriffe im 16. Jahrhundert? Mir scheint es naheliegender, von einem fühlbaren Körper zu sprechen. Palpabile, fühlbar, ist ein äußerst selten vorkommender Begriff in Vasaris Viten. Er verwendet ihn nur drei oder vier Mal: beispielsweise in der Paragone-Diskussion im Proemio der ersten Edition (1550), in dem es heißt, dass "allo scultore basta aver notizia delle vere forme e fattezze de' corpi solidi e palpabili e sottoposti in tutto al tatto [...] [mentre] al pittore è necessario non solo conoscere le forme di tutti i corpi retti e non retti, ma di tutti i trasparenti et impalpabili. ${ }^{23}$ („Dem Bildhauer genügt die Kenntnis der wirklichen Formen und der Beschaffenheit der festen, tastbaren [palpabile] und ganz dem Tastsinn unterworfenen Körper [...]. Für den Maler besteht nicht nur die Notwendigkeit, die Form aller gradlinigen 


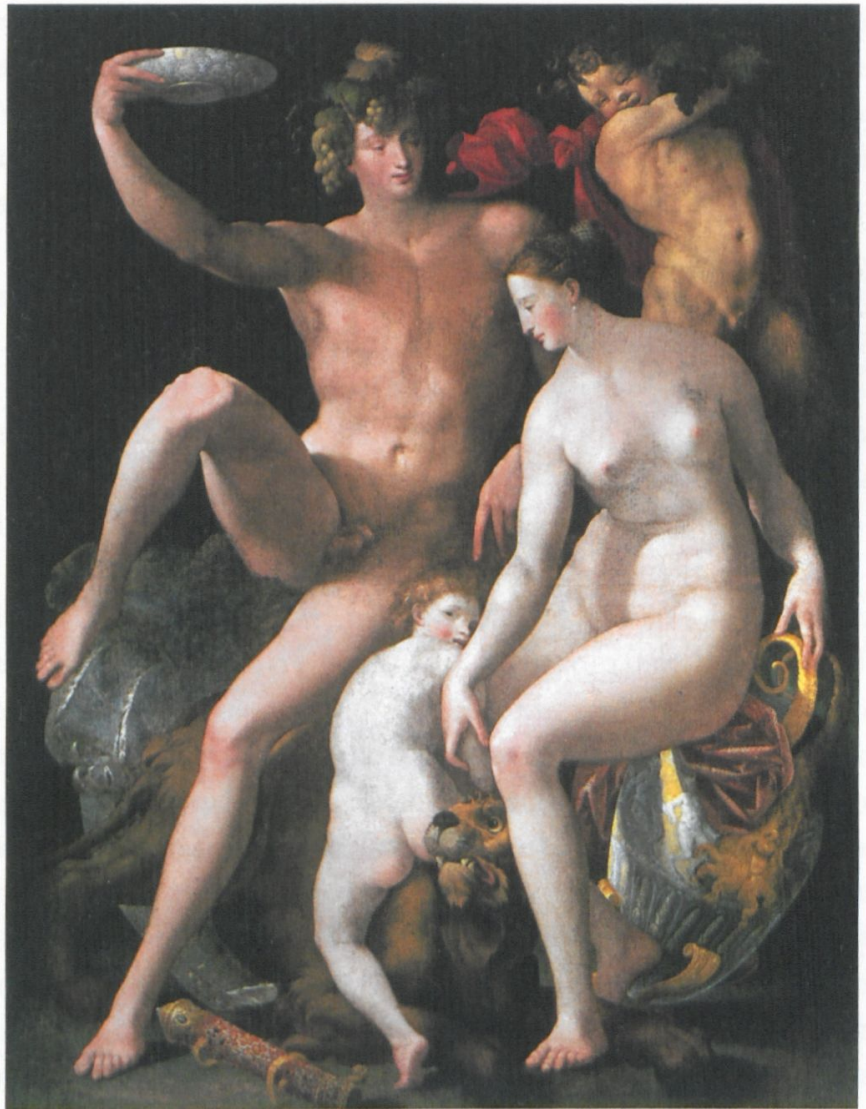

Bild 8 Rosso Fiorentino (Kopie nach): Venus und Bacchus, 1532, $209 \times 161,5 \mathrm{~cm}$, Musée national d'art et d'histoire, Luxembourg.

und nicht geraden Körper zu kennen, sondern auch diejenige aller durchsichtigen und nicht berührbaren. ${ }^{24}$ )

Vasari benutzt den Begriff ein weiteres Mal in der Vita Rossos. Doch bezieht er sich dabei nicht auf den von Engeln gestützten toten Christus, sondern auf das Bild von Bacchus, Venus und Amor (Bild 8), das sich einst in der Galerie von Fontainebleau befand und uns nur durch eine Kopie im Nationalmuseum von Luxemburg bekannt ist.

Die Tendenz, Kunstwerke durch eine Gegenüberstellung von plastisch Fühlbarem mit visuell Erfahrbarem zu erörtern, war schon seit Mitte des Quat-

Giorgio Vasari: Kunstgeschichte und Kunsttheorie. Eine Einführung in die Lebensbeschreibung berühmter Künstler, neu übers. v. Victoria Lorini, hg., eingel. u. komm. v. Matteo Burioni/Sabine Feser, Berlin 2004, S. 51. 
trocento weit verbreitet ${ }^{25}$ und wurde in die Diskussionen über den paragone aufgenommen. In diesen trat die Skulptur als natürliches Vehikel erotischer Darstellungen in Erscheinung, da der Tastsinn in enger Verbindung mit der Wollust gedeutet wurde. ${ }^{26}$ Aus diesem Grund scheinen mir die statuarische Qualität des von Rosso gemalten Körpers (nicht seine vollkommene Nacktheit) und die Geste des Engels, der als Metapher für den Tastsinn die Seitenwunde berührt, darauf zu verweisen, dass das, was wir als sinnlich wahrnehmen, trotz unterschiedlicher Konventionen auch damals ähnlich verstanden worden sein könnte. Wenn wir beim Anblick eines derart schönen und völlig nackten Körpers in einem sakralen Zusammenhang in Verwirrung geraten, müssen die Zeitgenossen Rossos, auch wenn sie in der Verherrlichung der nach antiken Modellen gestalteten Schönheit einen möglichen Weg zum christlichen Heil sahen, ebenfalls begriffen haben, was die taktilen Qualitäten des Werks implizierten.

Dennoch ist es nur den Engeln und den Heiligen gestattet, den Körper Christi zu berühren oder zu umarmen. Uns hingegen bleibt nur die Enthaltung, zu der schon Maria Magdalena mit den Worten „noli me tangere“ angehalten wurde, wenn wir nicht in Idolatrie verfallen wollen. Doch Rosso Fiorentino ist es wie kaum einem anderen gelungen, den Körper Christi physisch so präsent wiederzugeben und gleichzeitig die Spannung zwischen Tast- und Sehsinn zu thematisieren - zu einer Zeit, als der Sehsinn im Begriff war, sich gleichberechtigt neben jenem privilegierten erotischen Sinn zu etablieren. Daher scheint die Frage legitim, ob das aus der Verbreitung einer neuen bildlichen Laienkultur resultierende Klima bei der druckgraphischen Reproduktion nicht in irgendeiner Weise, wenn auch indirekt, dazu beigetragen haben könnte, im sakralen Bereich eine solche sinnliche Atmosphäre zu erzeugen?

Freilich ist es nicht erlaubt, Sakrales mit Heiligem zu vermischen, die körperliche Schönheit mit jener himmlischen und ewigen zu verwechseln. Trotzdem deuten die Texte dieser Epoche die Möglichkeit von Mißverständnissen an, nicht nur auf der Rezeptionsebene, sondern auch im Bereich der Produktion. Während Mario Equicola im zweiten Teil seines 1526 in endgültiger Fassung erschienenen Libro di natura d'amore die Wahrnehmung des wahren Philosophen, der sich der göttlichen Kontemplation von Schönheit hingibt, von der Wahrnehmung der lasterhaften und unzüchtigen Menschen unterscheidet, die von den Mysterien der Religion keine Kenntnis haben, verdammt Erasmus and Gender in Early Modern Europe, Cambridge 1993, S. 55-89, S. 62.

26 Wie es die explanatio von Jodocus Badius Ascensius in der französischen Übersetzung des Narrenschiffs von Sebastian Brant bestätigt; siehe Carl Nordenfalk: The Five Senses in Late Medieval and Renaissance Art, in: Journal of the Warburg and Courtald Institutes 48 (1985), S. 10-15. 
in seinem 1528 gedruckten Ciceronianus explizit jene Künstler, die Christus wie einen Apoll darstellen.

Was das schwierige Verhältnis von bellezza und decoro angeht, bleibt Vasaris Position exemplarisch, die er zu Beginn der Vita Fra Angelicos in der Edition von 1550 formuliert:

"Zweifellos sollte jemand, der geistliche und fromme Werke erschafft, auch stets geistlichen Standes und fromm sein, denn wenn sie von Personen gestaltet sind, die wenig Glauben besitzen und die Religion wenig schätzen, sieht man, daß sie häufig unehrenhafte Gelüste und wollüstige Begierden wecken [....]. Ich möchte jedoch nicht, daß manche sich schon dazu verleiten lassen, den Frommen als geschmacklos und unpassend zu interpretieren, wie es gewisse Leute tun, die beim Anblick von Gemälden, in denen eine Figur, sei es eine Frau oder ein Jüngling, ein wenig liebreizender, schöner und anmutiger als sonst üblich dargestellt ist, diese sofort als lasziv ansehen und beurteilen. ${ }^{27}$

Dieses Konzept wird in einem weniger beachteten Passus der 1568er-Ausgabe wieder aufgegriffen: „Giovan Francesco Caroto war beim Antwortgeben überaus geistvoll; so erzählt man sich auch, daß zu der Zeit, als er an einem Tag von einem Priester gesagt bekam, seine Altarfiguren seien zu lasziv, er zur Antwort gab: Ihr seid verloren, wenn die gemalten Dinge Euch erregen: Bedenkt wie man Euch Vertrauen schenken soll, wenn lebendige und tastbare [palpabile] Personen da sind. ${ }^{228}$ (Dies ist neben den beiden oben genannten Abschnitten der dritte Passus der Vite, in dem Vasari den Begriff „palpabile“ gebraucht).

Der Begriff „palpabile" bedeutet also „wirklich”, „real”, und Vasari verwendet ihn signifikanterweise sowohl zur Beschreibung von Qualitäten der Skulptur als auch für ein Gemälde Rossos. Rossos lebendiger und fühlbarer Christus war im herkömmlichen Sinne des Wortes, wie es im Cinquecento verstanden wurde, gewiss nicht "lasziv", doch zeigen die Zeilen des Erasmus und jene Vasaris wie eine solch ungestüme Fleischlichkeit hätte missverstanden werden können, zumindest von denjenigen, die mit den körperlichen Augen des Pöbels urteilen, um Leone Ebreos Worte zu gebrauchen. ${ }^{29}$

Ich möchte deshalb zwischen zwei Positionen vermitteln, die nur dem Anschein nach unversöhnlich sind: Zwischen der Auffassung derjenigen, die explizit die sinnliche Ambivalenz dieses Körpers hervorgehoben haben, ${ }^{30}$ und der Auffassung jener, die sich einzig und allein auf den theologischen Filter und 
die visuellen Codes verlassen haben, die wie die Antike kulturell akzeptiert waren, und sich dabei weigerten, den Unruhe stiftenden Charakter des Gemäldes anzuerkennen. (Und bei dieser Gelegenheit muss man betonen, dass die taktilen Qualitäten des Bildes seinerzeit auch Missbehagen hervorgerufen haben müssen).

In Wirklichkeit kann das Bild auf zwei Ebenen gelesen werden: als bewusste und orthodoxe theologische Botschaft von Gott, der für unsere Erlösung Mensch wurde, und als unbewusste Manifestation einer unlösbaren Spannung zwischen einer sakralen Utopie, die einen Kompromiss mit den Modellen der klassischen (fühlbaren) Schönheit anstrebt, und den optischen Anregungen einer neuen bildlichen Laienkultur, die sich für nackte Körper begeisterte. Mit anderen Worten, wir müssen zwischen Produktion und Rezeption differenzieren: Auch wenn der Christus in forma Pietatis im Hinblick auf die Darstellung eines sublimen christlichen Glaubens ein vollkommenes Werk ist, und das verzückte Antlitz des Erlösers dazu dient, die geistige Liebe Jesu, der sich für uns geopfert hat, bewusst zu vermitteln, bleibt das Bild auf der Rezeptionsebene dennoch unlösbar, weil es in sich die Spuren des unüberwindbaren Konflikts zwischen dem Wunsch zur Berührung (Thomas) und der diesbezüglichen Untersagung (Maria Magdalena) trägt, zwischen dem Blick jener, die mit den körperlichen Augen des Pöbels urteilen, und jener, die mit klaren Augen urteilen und „viel mehr als jene mit körperlichen Augen sehen".

Wenn Rosso das Werk in ein Umfeld entlässt, das nicht mehr seines und nicht länger kontrollierbar ist, ist dies der Augenblick, in dem sich die unbewussten Widersprüche manifestieren, in dem die rein christliche Absicht den Zwiespalt der Entstehung aufdeckt. Das Werk ist nicht blasphemisch, da die Intention zweifellos „rein christlich" war, doch kreiert es eine Beklommenheit, weil es versucht, zwei separate Welten in einem sakralen Kontext in Einklang zu bringen: in diesem Bild haben sich indirekt die neuen Konventionen der Sinnlichkeit eingeschlichen. Wie Erasmus ein Jahr zuvor in seinem Ciceroniano schrieb: Es ist nicht erlaubt, Gottvater im Gewand Jupiters zu malen, noch Christus als Apoll "quia signa rebus non congruent ${ }^{\prime \prime 31}$, denn dieses Aussehen wäre der Bedeutung der Person nicht angemessen. Dass der von Rosso und seinem Auftraggeber eingeschlagene Weg nicht begehbar war, wird durch den Umstand bezeugt, dass die kühne Erfindung des Künstlers, jedenfalls soweit mir bekannt ist, niemals weder kopiert noch wiederholt wurde.

we possess of religiously as well as morally cynical attitudes within élite circles of contemporary Rome." 